\title{
Structural and optoelectronic properties of $\mathrm{CsSnBr}_{3}$ metal halide perovskite as promising materials toward novel-generation optoelectronics
}

\author{
M. M. Saad ${ }^{\mathrm{a}, *}$, S. E. A. Yousif ${ }^{\mathrm{b}}$ \\ ${ }^{a}$ Department of Physics, College of Science and Arts in Al-Muthnib, Qassim \\ University, Al-Muthnib 51931, Saudi Arabia. \\ ${ }^{b}$ Department of Physics, Faculty of Science and Arts in Baljurashi, Albaha \\ University, Baljurashi1998, Saudi Arabia
}

\begin{abstract}
In this study, first-principles density functional theory (DFT) calculations of the structural and optoelectronic properties of Sn-based inorganic metal halide perovskite $\mathrm{CsSnBr}_{3}$ are carried out and discussed in details. The Wu-Cohen (WC)-Generalized Gradient Approximation (GGA) based on the full-potential linearized augmented plane-wave (FPLAPW) method is used to optimize the geometry structure of unit cell and then find the accurate optoelectronic properties of $\mathrm{CsSnBr}_{3}$. Analysis of structural optimization results revealed that the lattice parameters $\left(a_{0}=5.776 \AA\right)$ and unit cell volume of $\mathrm{CsSnBr}_{3}$ are exactly consistent with the experiments reports. Based on the results of band structures and density of states, $\mathrm{CsSnBr}_{3}$ is found to be nonmagnetic semiconductor with suitable direct band gap of $\left(\mathrm{E}_{\mathrm{g}}=0.610 \mathrm{eV}\right)$ along the $\mathrm{R}$ symmetry point. In addition, the calculations of optical properties of $\mathrm{CsSnBr}_{3}$, such as the real $\varepsilon_{1}(\omega)$ and imaginary $\varepsilon_{2}(\omega)$ parts of the dielectric function, $\varepsilon(\omega)$, absorption coefficient $\alpha(\omega)$, reflectivity $R(\omega)$ and refractive index $n(\omega)$, have been performed in the photonic energy range of $(0.0-15.0 \mathrm{eV})$. Finally, the results attained in the present study, which include the stable crystal structure and the high accurate optoelectronic properties such as appropriate direct band gap and high absorption of visible radiation, confirm the possible utilization of $\mathrm{CsSnBr}_{3}$ materials in novel optoelectronics applications as photovoltaic solar cells, photosensors, photodetectors, photodiodes and other related optoelectronics devices.
\end{abstract}

(Received November 14, 2021; Accepted February 26, 2022)

Keywords: Inorganic materials, Metal halide perovskites, Semiconductors, Optoelectronics, WC method

\section{Introduction}

In the last few years, the distinctive family of halide perovskite solar cells (HPSCs) with an exclusive chemical formula $\left(\mathrm{ABX}_{3}\right)$ have attracted extreme interest as excellent spectra absorbers for photovoltaics and other novel optoelectronics applications. According to the sort of ion or element that occupies the A-site, the $\mathrm{ABX}_{3}$ family can be classified into two famous classes of halide compounds: (i) organic-inorganic halide perovskites and (ii) all-inorganic halide perovskites. The crystal of the first class contains organic ions $[\mathrm{A}=$ Methylammonium (MA), $\left(\mathrm{CH}_{3} \mathrm{NH}_{3}\right) \mathrm{BX}_{3}$; Formamidinium (FA), $\left(\mathrm{HC}\left(\mathrm{NH}_{2}\right)_{2} \mathrm{BX}_{3}\right.$ ]. While, in all-inorganic halide perovskites, A-site is often occupied by a metallic element $(\mathrm{A}=\mathrm{Metal})$. The second class is considered one of most studied compounds during the current era, where various all inorganic HPSCs with $\mathrm{ABX}_{3}$ (A $=\mathrm{K}, \mathrm{Rb}, \mathrm{Cs} ; \mathrm{B}=$ Metal; $\mathrm{X}=$ Halogen) have been created and investigated. Besides studying their unique characteristics like flexible crystal structure, tunable semiconducting band-gap $\left(\mathrm{E}_{\mathrm{g}}<2.5\right.$ eV) [1-3], high charge-carrier mobility, large optical absorption coefficient and high composition stability [4], research also seek for promising and multi-faceted thermal, electrical and optical applications that give an extraordinary power-conversion efficiency $(\sim 24.0 \%)$ in photovoltaic solar cells technologies [5-12].

Photovoltaic solar cells are novel devices utilized mainly to convert the free solar radiation into an electrical current through the photovoltaic effect. These devices present an optoelectronics

\footnotetext{
*Corresponding author: $141261 @$ qu.edu.sa https://doi.org/10.15251/CL.2022.192.153
} 
design based of the combination of two semiconductor slabs with different electron concentration $[4,8]$. As shown in (Fig. 1) these slabs build mainly from two different electrical materials that can be characterized as N-type semiconductor material owns an excess of negative charges, i.e. electrons, and P-type semiconductor material having an excess of positive charges, i.e. holes. As a result, when both N-type and P-type slabs are in contact, some energetic electrons flow from the $\mathrm{N}$-type slab to the P-type slab, and some energetic-holes flow conversely from the P-type slab to the N-type slab, which produce a diffusion current through the P-N junction. Moreover, the fixed ions near the two limits of P-N junction generate an electric field in the opposite direction to the diffusion of these charges, which leads to the generation of drift current. As it is well known that the potential barriers establish at the P-N junction as a result of the balance between this drift current and diffusion current at equilibrium, which make the net current that flows through this structure equal to zero [7,8]. When the incidents solar radiation strikes the surface of solar cell, the $\mathrm{N}$-type electrons absorb the energy carried by its photons, which breaks their chemical bonds and creating electron-hole pairs in the N-type material. These two charge carriers are pushed up by the electric field, causing them to flow through the P-N junction solar cell, and if a load is connected, an electric potential difference between the terminals of solar cell will be established, which generates an electric current flowing through the external electrical load in the outer circuit.

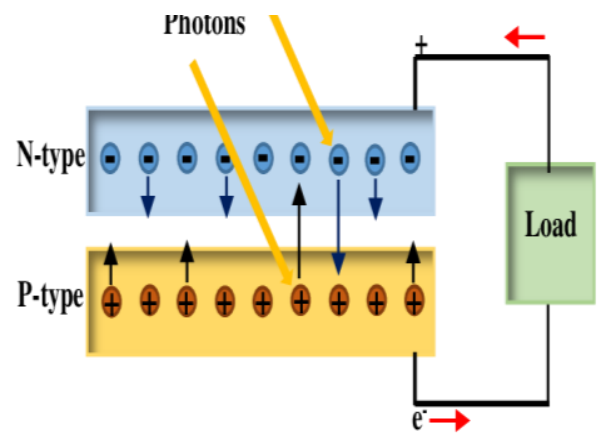

Fig. 1. The photovoltaic effect and schematic illustration of $P-N$ junction solar cell.

Therefore, the current challenge is to synthesis $\mathrm{ABX}_{3}$ solid materials provide suitable properties, include notable chemical stability at high temperatures, high electrical power conversion efficiency, broad emission and tunable semiconducting $\mathrm{E}_{\mathrm{g}}$ [13]. Motivated by the effect of $\mathrm{A}$ - and $\mathrm{B}$-site substitution in $\mathrm{ABX}_{3}$ unit cell, we extended this concept to produce a promising compound of $\mathrm{CsSnBr}_{3}$ and explore its structural, and optoelectronic properties. Recently, several research studies have been done on numerous cubic compounds within $\mathrm{ABX}_{3}$ family, which is the most common structure in materials science and solid state physics. Due to the possibility of substituting the three positions $\mathrm{A}, \mathrm{B}$ and $\mathrm{X}$ in $\mathrm{ABX}_{3}$ by suitable metallic elements and halogens, respectively, these compounds can have all potentials to become promising materials with multifunctional properties [14-16]. Thus, most $\mathrm{ABX}_{3}$ compounds can have remarkable performances in many fields of future applications, such as in photovoltaic solar cells (Fig. 1) [17], memories, sensors and diode devices [3,7], electrodes [15], as well as in ferroelectricity, superconductivity and piezoelectricity applications [14]. These physical properties have attracted widespread attention of scientists, engineers and researchers to devote enormous efforts on these $\mathrm{ABX}_{3}$ materials to investigate and detect novel and useful properties for different future applications $[1,8,11,14]$.

In this work, in order to contribute and develop the materials research in this field as well as to explore new properties among the HPSC compounds that are not yet predicted experimentally or theoretically, we have intended to investigate the main physical properties of $\mathrm{ABX}_{3}$ by choosing its sites as: $\mathrm{A}$ is an alkali-metal, i.e. Cesium atom $(\mathrm{A}=\mathrm{Cs}), \mathrm{B}$ is post-transition metal $(B=S n)$ and $X$ is a halogen element $(X=B r)$. This study proves a distinguished contribution in this field by examining the effect of site substitution $(A=C s, B=S n)$ and applying WC method on the structural and optoelectronic properties of $\mathrm{ABX}_{3}$ compared to the published 
properties of related halide perovskites. The main goal of this study, is to provide GGA theoretical basses to realize all these physical properties of cesium bromide $\mathrm{CsSnBr}_{3}$ and their dependence on the type of B-site. All these calculations were carried out by utilizing the WC method based on the Generalized Gradient Approximation (GGA), to well examine the structural, electronic and optical properties of $\mathrm{CsSnBr}_{3}$. As a result of this work and based on their obtained chemical and physical properties, we can expect the possible applications of $\mathrm{CsSnBr}_{3}$ halide perovskites in photovoltaic solar cells, photodetectors, photodiodes and other related optoelectronics.

\section{Computational methods}

The ground state properties of $\mathrm{CsSnBr}_{3}$ are calculated using the first-principles density functional theory (DFT) [18], as executed in the WIEN2k [19] package. Full-potential linearized augmented plane-wave (FP-LAPW) method is utilized. The Wu-Cohen (WC) [18-20] method based on the Generalized Gradient Approximation (GGA) [21] is implemented to modulate the structural and optoelectronic properties of this compound. Non spin-polarized version of the WCGGA calculations are performed to obtain the appropriate results of these properties.

In PFLAPW method, the unit cell of $\mathrm{CsSnBr}_{3}$ contains two main regions; the first of which is a spherical region, muffin-tin (MT), where the potential is expected to be spherically symmetric, this region is definite by $\left(r \leq R_{M T}\right)$ where $R_{M T}$ is the muffin-tin radius. In all WC-GGA calculations, the $\mathrm{R}_{\mathrm{MT}}$ values of the individual elements in $\mathrm{CsSnBr}_{3}$ are set as $\left[\mathrm{R}_{\mathrm{MT}}(\mathrm{Cs})=2.2, \mathrm{R}_{\mathrm{MT}}\right.$ $(\mathrm{Sn})=2.0$ and $\mathrm{R}_{\mathrm{MT}}(\mathrm{Br})=1.8$ a.u.]. In the MT region, the solution of the Schrödinger equation is given by a radial function times a spherical harmonic, whereas in the interstitial region (IR), the potential is considered constant and the Schrödinger equation is solved by plane wave functions.

The ground state energy convergence to $(0.00001 \mathrm{Ry})$ was achieved using the total number of (k-points $=2000)$ in the first Brillouin zone $(B Z)$ with a cutoff value $\left(\mathrm{R}_{\mathrm{MT}} \mathrm{K}_{\max }=8.0\right)$, where $\mathrm{R}_{\mathrm{MT}}$ corresponds to smallest MT radius and $\mathrm{K}_{\max }$ is the largest reciprocal lattice vector [19]. The structural optimizations of $\mathrm{CsSnBr}_{3}$ were carried out as a first stage, where the unit cell energy has been varied with respect to its unit cell volume.

\section{Results and discussion}

As an initial utilization of the skills provided by the WIEN2k code, the optimized structural parameters of perovskite $\mathrm{CsSnBr}_{3}$ are obtained from the Energy vs. Volume calculation in nonmagnetic (NM) and ferromagnetic (FM) phases . Fig. 2 show the predicted results of structural optimization by fitting the variation of the total energy per unit cell of $\mathrm{CsNBr}_{3}$ as a function of its corresponding volume by means of the Murnaghan equation of state:

$$
E-E_{0}=\frac{B_{0} V}{B_{0}^{\prime}}\left[\frac{\left(V_{0} / V\right)^{B_{0}^{\prime}}}{\left(B_{0}^{\prime}-1\right)}+1\right]-\left[\frac{B_{0} V_{0}}{\left(B_{0}^{\prime}-1\right)}\right]
$$

where, $E=$ the total energy per unit cell of $\mathrm{CsNBr}_{3}, E_{0}=$ the ground state energy, $E=$ the unit cell volume of $\mathrm{CsNBr}_{3}, V_{0}=$ the equilibrium unit cell volume with lattice constants of $\left(a_{0}=b_{0}=\right.$ $\left.c_{0}\right), B_{0}=$ the bulk modulus, and $B_{0}^{\prime}=$ the pressure derivative of bulk modulus.

It is clearly that the crystal structure of $\mathrm{CsSnBr}_{3}$ in NM phase is more stable than in FM phase because it shows less energy $\left(E_{N M}<E_{F M}\right)$. Table 1 summarizes the structural data of $\mathrm{CsSnBr}_{3}$ extracted from the optimization calculations compared with the available experimental and previous DFT results. 


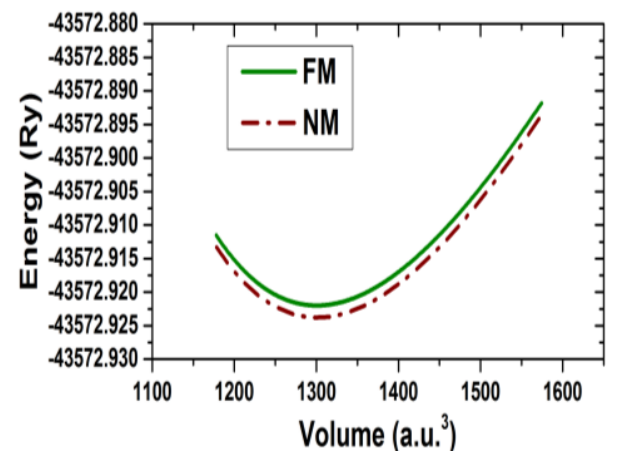

Fig. 2. The optimized of energy vs. unit cell volume of metal halide perovskite CsSnBr$r_{3}$.

Table 1. The optimized structural data of metal halide perovskite CsSnBr 3 .

\begin{tabular}{|l|l|l|}
\hline Parameter & Present & Other $[25,26]$ \\
\hline S. G. & Pm-3m & Pm-3m \\
\hline$a_{0}(\AA)$ & 5.776 & $5.883,5.800$ \\
\hline$V_{0}\left(\AA^{3}\right)$ & 192.7 & \\
\hline$B_{0}(\mathrm{GPa})$ & 22.19 & \\
\hline$B_{0}^{\prime}(\mathrm{GPa})$ & 4.634 & \\
\hline $\mathrm{T}_{\mathrm{F}}$ & 1.006 & \\
\hline $\mathrm{Sn}-\mathrm{Br}(\AA)$ & 2.888 & \\
\hline$E_{0}(\mathrm{Ry})$ & -43572.92 & \\
\hline $\mathrm{E}_{\mathrm{g}}(\mathrm{eV})$ & 0.610 & $0.600,0.626$ \\
\hline
\end{tabular}

In view of these, it can conclude that the obtained value of $a_{0}$ is in a good agreement with those results. Also, the value of $B_{0}$ illustrates the mechanical stiffness of $\mathrm{CsSnBr}_{3}$ which makes it more rigid material and less compressible. Alike expectation was reported for analogous metal halide perovskites within GGA studies [22]. Also, the atomic distances and positions in the cubic unit cell of $\mathrm{CsSnBr}_{3}$ are shown in (Fig. 3), Cs at $1 \mathrm{a}(0,0,0)$, $\mathrm{Sn}$ at $1 \mathrm{~b}(0.5,0.5,0.5)$ and $\mathrm{Br}$ at $3 \mathrm{~d}$ $(0.5,0,0),(0,0.5,0)$ and $(0,0,0.5)$. Moreover, two important characteristics that determine the suitability of $\mathrm{CsSnBr}_{3}$ material for the manufacture of photovoltaics solar cells and related optoelectronics devices are quantum efficiency and chemical stability. Tolerance factor [23] evaluates the chemical stability of $\mathrm{CsSnBr}_{3}$ by means of ionic radii, as:

$$
T_{F}=\frac{0.71\left(r_{C S}+r_{B r}\right)}{\left(r_{S n}+r_{B r}\right)}
$$

where, $\left(\mathrm{r}_{\mathrm{CS}}=2.02 \AA\right)$ in XII-coordinate system, and $\left(\mathrm{r}_{\mathrm{Sn}}=0.89 \AA\right)$ and $\left(\mathrm{r}_{\mathrm{Br}}=1.82 \AA\right)$ in the VIcoordinate system [24]. As a result of applying this formula, it is found that the stable cubic crystal structure of halide perovskite $\mathrm{CsSnBr}_{3}$ gives a value close to the standard value $\left(\mathrm{T}_{\mathrm{F}} \approx 1.00\right)$, which matches also the (Pm-3m; \#221) symmetry. 


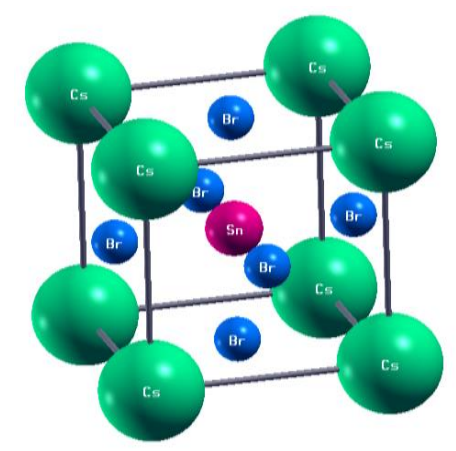

Fig. 3. The cubic crystal structure of metal halide perovskite CsSnBr 3 .

In order to study the electronic properties of $\mathrm{CsSnBr}_{3}$, its 2D charge density, band structure, total density of states (TDOS) and partial density of states (PDOS) have been calculated and discussed deeply as follow. First, Fig. 4 represents the charge density in (100) plane of the unit cell of $\mathrm{CsSnBr}_{3}$, which gives a clear picture of the chemical bonding nature between the three atoms $\mathrm{Cs}, \mathrm{Sn}$ and $\mathrm{Br}$ in their unit cell $\mathrm{CsSnBr}_{3}$ as well as allows to explain the charge transfer mechanisms through these bonds. It can be seen that there is formation of orbital hybridizations between $\mathrm{p}$-states of cations $\mathrm{Sn}-5 \mathrm{p}$ and anions $\mathrm{Br}-4 \mathrm{p}$. The condensed contour lines around $\mathrm{Sn}$ and $\mathrm{Br}$ indicates their covalent bond nature whereas the formation of other bonds $\mathrm{Cs}-\mathrm{Br}$ have an ionic nature with minor contour lines.
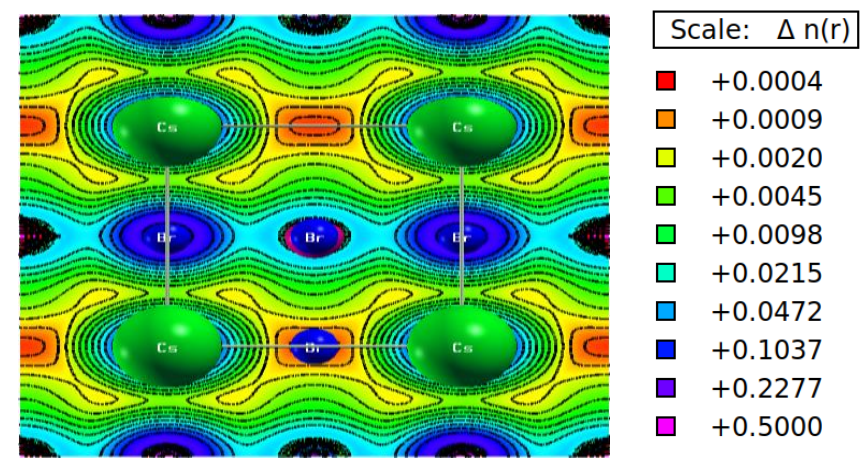

Fig. 4. The 2D charge density in (100) plane for the unit cells of metal halide perovskite CsSnBr 3 .

Second, an important parameter of semiconductor is the value of band gap $\left(\mathrm{E}_{\mathrm{g}}\right)$, can be extracted from the results of calculated band structures, which can be used to evaluate the potential of this compound for optoelectronics applications. Fig. 5 shows the calculated band structures of $\mathrm{CsSnBr}_{3}$ along their high cubic symmetry points within the first BZ. The key remark here, that perovskite $\mathrm{CsSnBr}_{3}$ shows semiconductor property with a direct $\mathrm{E}_{\mathrm{g}}$ at $\mathrm{R}$-point of about $\left(\mathrm{E}_{\mathrm{g}} \approx 0.610\right.$ $\mathrm{eV})$ in good agreement with the experimental value $\left(\mathrm{E}_{\mathrm{g}} \approx 0.600 \mathrm{eV}\right)$ and smaller than GGA result $\left(\mathrm{E}_{\mathrm{g}} \approx 0.626 \mathrm{eV}\right)[25,26]$. The indirect $\mathrm{E}_{\mathrm{g}}$ of $\mathrm{CsSnBr}_{3}$ locates between the valence band maxima and conduction band minima, which both lie along the $\mathrm{R}$ symmetry point. Where, the valence band maxima line touch the Fermi level $\left(\mathrm{E}_{\mathrm{F}}=0.0 \mathrm{eV}\right)$. Compared with its analogous $\mathrm{Ge}$ and $\mathrm{Pb}$ based halide perovskites [1,22], the narrow $\mathrm{E}_{\mathrm{g}}$ shown by the $\mathrm{CsSnBr}_{3}$ crystal increases its significance for P-N junction (Fig. 1) in optoelectronics devices and photovoltaic solar cells applications. 


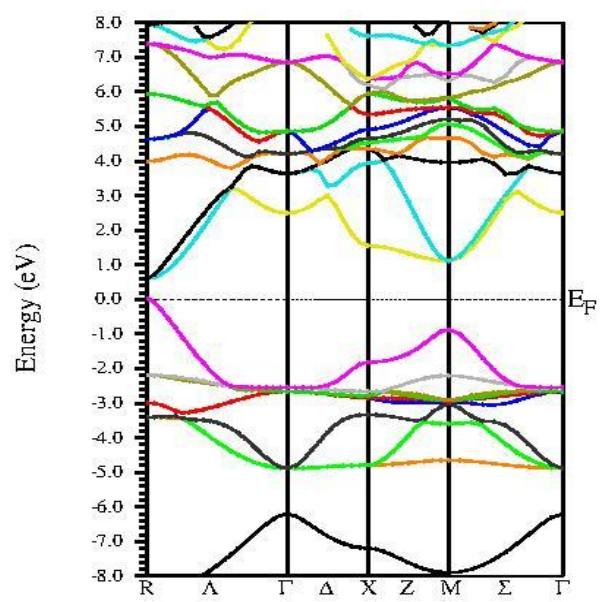

Fig. 5. The calculated band structures of metal halide perovskite $\mathrm{CsSnBr}_{3}$.

Third, in order to evaluate the atomic contribution to the density of states and nature of the atomic bonding in $\mathrm{CsSnBr}_{3}$, the total density of states (TDOS) and partial density of states (PDOS) are calculated using the WC method and shown in Figs. 6 and 7. It is obvious that TDOS (Fig. 6(a)) shows an $E_{g}$ at the $E_{F}$, which also predict the semiconducting behavior obtained from band structures. Also, the different contributions come from the TDOS per atom of $\mathrm{Cs}$, Sn and $\mathrm{Br}$ to the TDOS per unit cell of $\mathrm{CsSnBr}_{3}$ are plotted in (Fig. 6(b)).
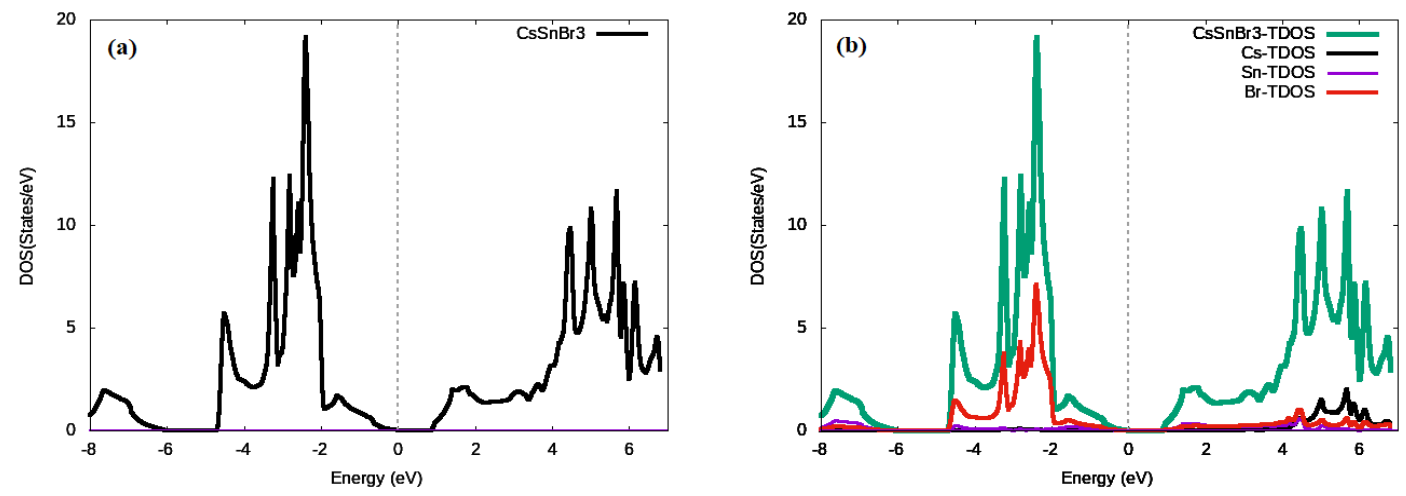

Fig. 6. The calculated total density of states; (a) TDOSs of CsSnBr $r_{3}$, and (b) TDOSs of Cs, Sn and Br of atoms, per unit

Moreover, the PDOSs per atom (Fig. 7(a,b, c)) confirm that the valence bands create from the partial hybridizations between $\mathrm{Sn}(5 \mathrm{~s}, 5 \mathrm{p})$ and $\mathrm{Br}(4 \mathrm{p})$ states extend between $-5.0 \mathrm{eV}$ and $\mathrm{E}_{\mathrm{F}}$, whereas the conduction bands are generally dominated also by the $\mathrm{Sn}(5 \mathrm{p})$ and $\mathrm{Br}(4 \mathrm{p})$ hybridizations. The top of valence bands is yielded by partial $5 s$ and $4 p$ states, $\mathrm{Sn}(5 \mathrm{~s})-\mathrm{Br}(4 \mathrm{p})$, and the bottom of conduction bands are occupied by $5 p$ and $4 p$ states with no contribution come from Cs (5p) and Cs (4d) states. The prediction of semiconducting property gives complete confidence of the tenability to develop such the proposed $\mathrm{CsSnBr}_{3}$ compound as promising candidate materials for novel light-emitting diodes, optoelectronic and photovoltaic technologies [27]. 

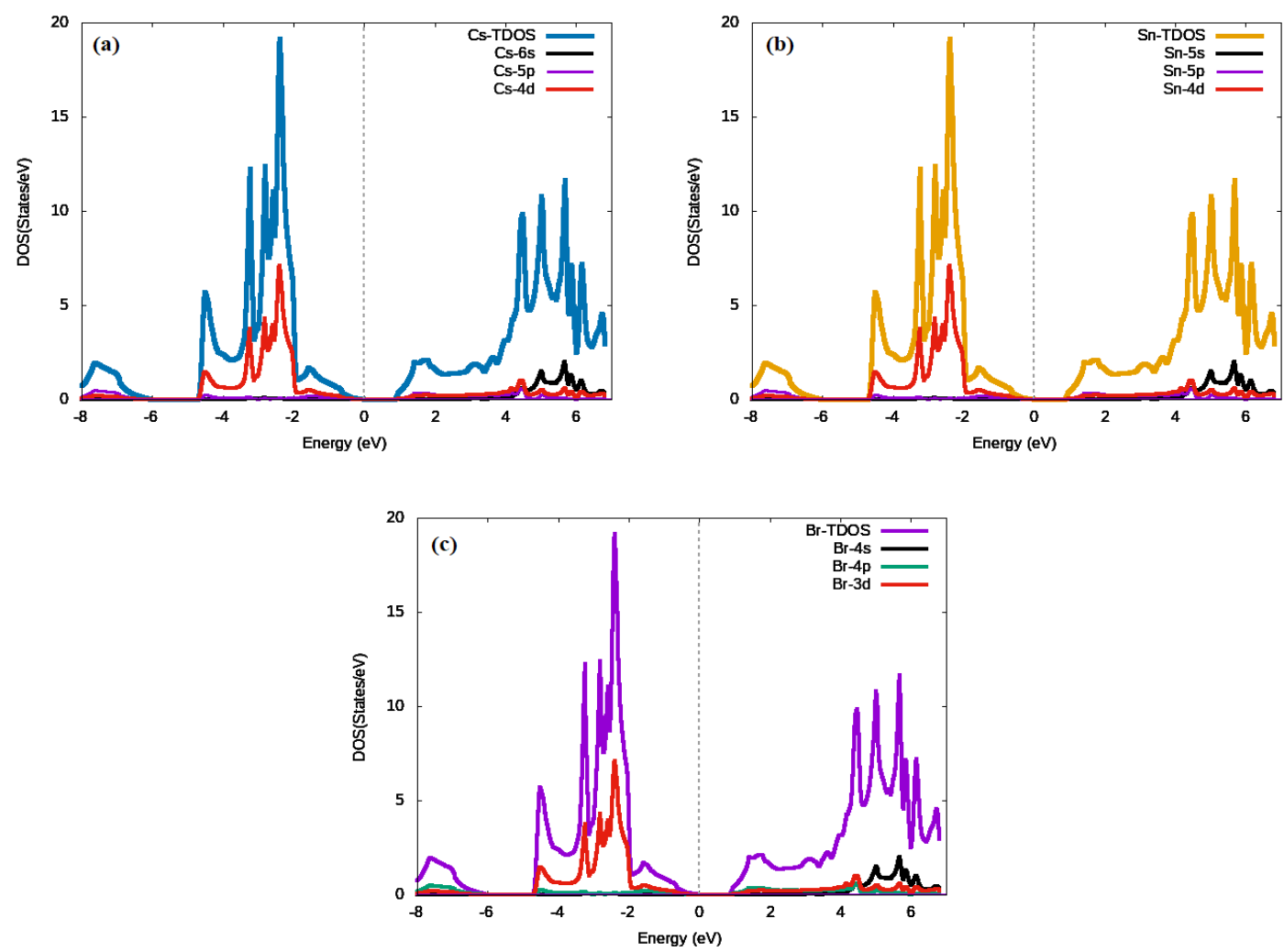

Fig. 7. The calculated partial density of states; PDOSs of (a) Cs, (b) Sn and (c) Br, per atom in metal halide perovskite $\mathrm{CsSnBr}$.

Studying of the optical properties allows us to determine the response of a certain material as a result of its interaction with the electromagnetic radiation. Thus, to examine this response, the key optical parameters of $\mathrm{CsSnBr}_{3}$ like real part $\varepsilon_{1}(\omega)$ of the dielectric function, the absorption coefficient $\alpha(\omega)$, the refractive index $n(\omega)$ and the reflectivity $R(\omega)$ are calculated and discussed in detail. The optical results obtained in the meaningful photonic energy range of $(0.0-15.0 \mathrm{eV})$ are plotted in Figs. 8 and 9.

First, from the $\varepsilon_{1}(\omega)$ curve (Fig. 8 (a)), which illustrates the degree of optical polarization of $\mathrm{CsSnBr}_{3}$ when the electromagnetic radiation interacts with its crystal, it can be seen that $\varepsilon_{1}(\omega)$ starts at zero energy $(\omega=0)$. This value corresponds to the electronic $\mathrm{E}_{\mathrm{g}}$ that defined by KramersKronig relations and Penn model [22]. $\varepsilon_{1}(0)-1=\left(\frac{E_{p}}{E_{g}}\right)^{2}$, where the plasma energy $\left(E_{p}=\hbar \omega_{p}\right)$, so, the narrow $\mathrm{E}_{\mathrm{g}}$ gives a larger value of its $\varepsilon_{1}(0)$ and verse versa. The static value of $\mathrm{CsSnBr}_{3}$ is $\left(\varepsilon_{1}(0)=6.74\right)$, which is in complete agreement with the conclusion drawn from the Penn's theoretical model. The $\varepsilon_{1}(\omega)$ curve increases to its first peak 7.09 at $0.42 \mathrm{eV}$ and maximum value 7.63 at $0.72 \mathrm{eV}$, then it reaches its negative value next $11.4 \mathrm{eV}$. This indicates that the electromagnetic radiation is reflected from the $\mathrm{CsSnBr}_{3}$ crystal and its photonic beam is totally reduced in this optical medium, making it as candidate materials for the protective shields from this radiation in the above specific energy range [25]. 

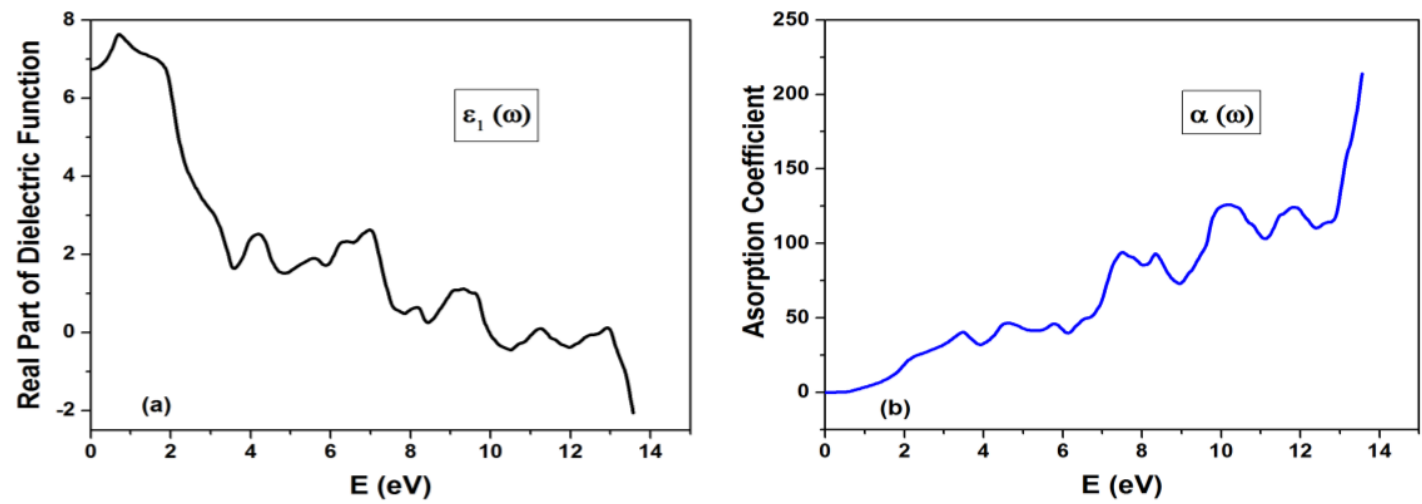

Fig. 8. The optical results of metal halide perovskite $C s \operatorname{SnBr}_{3}\left(\right.$ a) real part of dielectric function $\varepsilon_{1}(\omega)$, (b) absorption coefficient $\alpha(\omega)$, (c) refractive index $n(\omega)$ and $(d)$ reflectivity $R(\omega)$.

The absorption coefficient $\alpha(\omega)$ (Fig. 8 (b)), which describes the optoelectronic interaction between the incident photons and the electrons in crystal of $\mathrm{CsSnBr}_{3}$, thus, it can provide important evidence for the application prospects of this material. It is clear that $\alpha(\omega)$ curve increases with the increase of $\mathrm{E}(\mathrm{eV})$, through four main peaks at $4.42 \mathrm{eV}, 7.44 \mathrm{eV}, 10.11$ $\mathrm{eV}$ and $13.56 \mathrm{eV}$, in the visible range. $\mathrm{CsSnBr}_{3}$ shows high light absorption in the visible range due to its narrow $\mathrm{E}_{\mathrm{g}}$, which indicates that $\mathrm{CsSnBr}_{3}$ can be considered as suitable potential semiconductor crystal structures for many novel optoelectronics applications like photovoltaic solar cells, optical sensors and detectors [45]. The curve of reflectivity $R(\omega)$ for $\mathrm{CsSnBr}_{3}$ (Fig. 9 (a)) is consistent with the behavior of $\alpha(\omega)$, the static value is $(R(0) \approx 0.20)$ and its maximum value corresponds to the negative values of $\varepsilon_{1}(\omega)$ at $\mathrm{E}>11.4 \mathrm{eV}$, where the percentage of $R(\omega)$ is found to be $24.7 \%$. Finally, from the curve of refractive index $n(\omega)$ of $\mathrm{CsSnBr}_{3}$ in (Fig. 9 (b)), it is clearly seen that the static and calculated values of refractive index are equal, $n(0) \approx 2.60$, and $n(0)=\sqrt{\varepsilon_{1}(0)} \approx 2.596$, respectively.

Table 2 summarizes the calculated results and static values (Figs. 8 and 9) of the main optical parameters for the metal halide perovskite $\mathrm{CsSnBr}_{3}$ at zero energy $(E=0)$. These data include the maximum value real part of dielectric function $\varepsilon_{1}(0)$, the static value of reflective index $R(0)$, the static and calculated values of refractive index $n(0)$, and the evaluation rate of the optical absorption $\alpha(\omega)$.
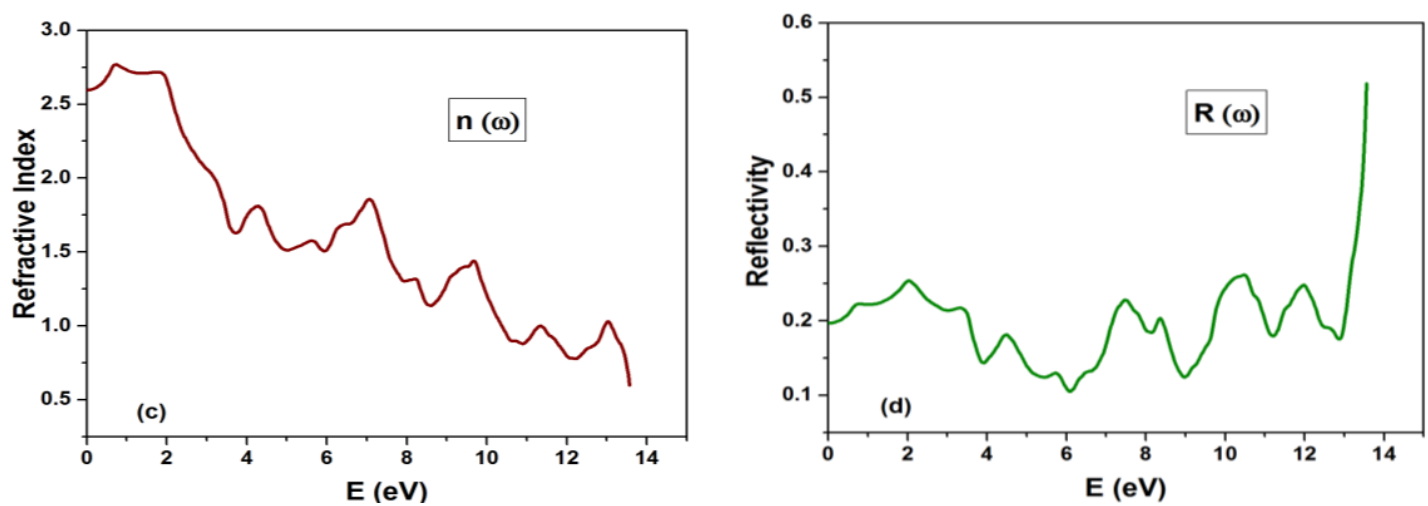

Fig. 9. The optical results of metal halide perovskite $C s \operatorname{SnBr}_{3}\left(\right.$ a) real part of dielectric function $\varepsilon_{1}(\omega),(b)$ absorption coefficient $\alpha(\omega)$, (c) refractive index $n(\omega)$ and $(d)$ reflectivity $R(\omega)$. 
Table 2. The main optical data with semiconductor nature of the metal halide perovskite CsSnBr $r_{3}$.

\begin{tabular}{|l|l|l|}
\hline Optical Parameter & Symbol & Value \\
\hline Maximum value of real part & $\varepsilon_{1}(0)$ & 6.740 \\
\hline Static reflective index & $R(0)$ & 0.200 \\
\hline Static refractive index & $n(0)$ & 2.600 \\
\hline Calculated refractive index & $n(0)=\sqrt{\varepsilon_{1}(0)}$ & 2.596 \\
\hline Absorption rate & $\alpha(\omega)$ & High \\
\hline
\end{tabular}

\section{Conclusions}

In this work, the structural, electronic and optical properties of all-inorganic metal halide perovskite $\mathrm{CsSnBr}_{3}$ were examined for novel optoelectronics applications by execution of density functional theory (DFT) based on the FP-LAPW-WIEN2k method. To highlight these properties, the WC method based on the Generalized Gradient Approximation (GGA) has been used in all calculations. The results obtained from structural optimizations that carried out using WC method, show that the compound $\mathrm{CsSnBr}_{3}$ stable in cubic structure (Pm-3m; no. 221) with ( $a_{0}=5.776 \AA$ ).

Also, the calculation results of band structures, density of states and 2D charge density show that the present perovskite $\mathrm{CsSnBr}_{3}$ exhibits nonmagnetic and semiconductor properties and characterized by an appropriate direct band gap of $\left(\mathrm{E}_{\mathrm{g}} \approx 0.610 \mathrm{eV}\right)$ lie along the R-R symmetry point. Moreover, the results obtained for the optical properties, the real part of the dielectric function, the absorption coefficient $\alpha(\omega)$, the refractive index $n(\omega)$ and the reflectivity $R(\omega)$, confirm that the crystal of $\mathrm{CsSnBr}_{3}$ is a good light absorber with high light absorption extends in the visible range.

Accordingly, the exclusive properties of $\mathrm{CsSnBr}_{3}$, that include high structural stability, high ductility and elasticity, suitable value of direct band gap and high optical absorption, make it a promising material for various novel applications. The present theoretical results provide a basis for the future experimental studies of Cs-based halide perovskites and related compounds, which have structural and optical properties may be necessary in novel fields of optoelectronics industries such as in photovoltaic solar cells, photodetectors, photodiodes and other photovoltaics devices.

\section{Acknowledgements}

Researchers would like to thank Deanship of Scientific Research, Qassim University and Albaha University, for motivating publish this paper.

\section{References}

[1] Y. Ye, X. Run, X. Hai-Tao, H. Feng, X. Fei, W. Lin-Jun, Chin. Phys. B 24(11), 116302 (2015).

[2] L. Sanchez, J. Antonio D. Pascual, A. M. Capilla, R. Pena, Int. J. Mol. Sci. 20, 976 (2019); https://doi.org/10.3390/ijms20040976

[3] Felix Deschler, Dieter Neher, Lukas Schmidt-Mende, APL Mater. 7, 080401 (2019); https://doi.org/10.1063/1.5119744

[4] A. K. Chilvery, A. K. Batra, B. Yang, K. Xiao, P. Guggilla, M. D. Aggarwal, R. Surabhi, R. B. Lal, J. R. Currie, B. G. Penn, J. Photon. Energy 5, 057402 (2015);

https://doi.org/10.1117/1.JPE.5.057402

[5] G. Lozano, J. Phys. Chem. Lett. 9(14), 3987 (2018);

https://doi.org/10.1021/acs.jpclett.8b01417

[6] M. Pazoki, T. Edvinsson, Sustainable Energy Fuels 2, 1430 (2018);

https://doi.org/10.1039/C8SE00143J

[7] S. Yun, Y. Qin, A. R. Uhl, N. Vlachopoulos, M. Yin, D. Li, X. Han, A. Hagfeldt, Energy 
Environ. Sci. 11, 476 (2018); https://doi.org/10.1039/C7EE03165C

[8] J. A. Luceno-Sanchez, A. M. Diez-Pascual, R. P. Capilla, Int. J. Mol. Sci. 20, 976 (2019); https://doi.org/10.3390/ijms20040976

[9] N. Kumar, J. Rani, R. Kurchania, Mater. Today-Proc. 46(11), 5570 (2021);

https://doi.org/10.1016/j.matpr.2020.09.349

[10] V. Jella, S. Ippili, J.-H. Eom, S.V.N. Pammi, J.-S. Jung, V.-D. Tran, V. H. Nguyen, A.

Kirakosyan, S. Yun, D. Kim, M. R. Sihn, J. Choi, Y.-J. Kim, H.-J. Kim, S.-G. Yoon, Nano Energy 57, 74 (2019); https://doi.org/10.1016/j.nanoen.2018.12.038

[11] W. Xiang, W. Tress, Advanced Materials 31(44), 1902851 (2019);

https://doi.org/10.1002/adma.201902851

[12] Q. Zhang, Y. Yin, ACS Cent. Sci. 4(6), 668 (2018);

https://doi.org/10.1021/acscentsci.8b00201

[13] D. B. Raja, K. S. Sundaram, R. Vidya, Solar Energy 207, 1348 (2020);

https://doi.org/10.1016/j.solener.2020.07.044

[14] L. M. Herz, ACS Energy Lett. 2(7), 1539 (2017);

https://doi.org/10.1021/acsenergylett.7b00276

[15] T. Ibn-Mohammed, S. C. L. Koh, I.M. Reaney, A. Acquaye, G. Schileo, K. B. Mustapha, R.

Greenough, Renew. Sust. Energy Rev. 80, 1321 (2017); https://doi.org/10.1016/j.rser.2017.05.095

[16] A. Zhang, Y. Chen, J. Yan, IEEE J. Quantum Elect. 52(6), 1600106 (2016);

https://doi.org/10.1109/JQE.2016.2563783

[17] Z. Shi, A. H. Jayatissa, Materials 11(5), 729 (2018); https://doi.org/10.3390/ma11050729

[18] P. Hohenberg, W. Kohn, Phys. Rev. 136(3B), 864 (1964);

https://doi.org/10.1103/PhysRev.136.B864

[19] K. H. Schwarz, P. Blaha, G. K. H. Madsen, Comput. Phys. Commun. 147, 71 (2002);

https://doi.org/10.1016/S0010-4655(02)00206-0

[20] J. P. Perdew, K. Burke, M. Ernzerhof, Phys. Rev. Lett. 77, 3865 (1996);

https://doi.org/10.1103/PhysRevLett.77.3865

[21] Z. Wu, R. E. Cohen, Phys. Rev. B 73, 235116 (2006);

https://doi.org/10.1103/PhysRevB.73.235116

[22] M. Houari, B. Bouadjemi, S. Haid, M. Matougui, T. Lantri, Z. Aziz, S. Bentata, B. Bouhafs, Indian J. Phys. 94(4), 455 (2020); https://doi.org/10.1007/s12648-019-01480-0

[23] V. M. Goldschmidt, Die Gesetze der Krystallochemie. D. Naturwiss. 14, 477 (1926); https://doi.org/10.1007/BF01507527

[24] R. D. Shannon, Acta Cryst. A 32, 751 (1976); https://doi.org/10.1107/S0567739476001551

[25] M. A. Islam, Md. Zahidur Rahaman, Sapan Kumar Sen, AIP Adv. 11, 075109 (2021); https://doi.org/10.1063/5.0057287

[26] W.-J. Shi, J. Liu, Y. Xu, S.-J. Xiong, J. Wu, W. Duan, Phys. Rev. B 92, 205118 (2015);

https://doi.org/10.1103/PhysRevB.92.205118

[27] K. Ji, M. Anaya, A. Abfalterer, S. D. Stranks, Adv. Optical Mater., 2002128 (2021); https://doi.org/10.1002/adom.202002128

[28] D. Menzel, A. Tejada, A. Al-Ashouri, I. Levine, J. A. Guerra, B. Rech, S. Albrecht, L. Korte, ACS Appl. Mater. Interfaces 13(36), 43540 (2021); https://doi.org/10.1021/acsami.1c10171 